See discussions, stats, and author profiles for this publication at: https://www.researchgate.net/publication/301365459

\title{
Rheological investigation of highly filled polymers: Effect of molecular weight
}

Conference Paper · April 2015

DOI: $10.1063 / 1.4918891$

READS

3

5 authors, including:

Eva Hnátková

Tomas Bata University in Zlín

11 PUBLICATIONS 3 CITATIONS

SEE PROFILE
Berenika Hausnerova

Tomas Bata University in Zlín

88 PUBLICATIONS 320 CITATIONS

SEE PROFILE 


\title{
Rheological Investigation of Highly Filled Polymers: Effect of Molecular Weight
}

\author{
Eva Hnatkova ${ }^{\mathrm{a}, \mathrm{b}}$, Andrew Hales ${ }^{\mathrm{c}}$, Lukas Jiranek ${ }^{\mathrm{d}}$, Juan Miguel Alcon \\ Vera $^{\mathrm{e}}$, Berenika Hausnerová ${ }^{\mathrm{a}, \mathrm{b}}$ \\ ${ }^{a}$ Department of Production Engineering, Faculty of Technology, Tomas Bata University in Zlin, \\ nám. T. G. Masaryka 5555, 76001 Zlín, Czech Republic \\ ${ }^{b}$ Centre of Polymer Systems, University Institute, Tomas Bata University in Zlin, \\ Nad Ovčírnou 3685, 76001 Zlín, Czech Republic \\ ${ }^{c}$ The University of Sheffield, Department of Materials Science and Engineering, Sir Robert Hadfield \\ Building, Mappin Street, Sheffield, S1 3JD, United Kingdom \\ ${ }^{d}$ Beckett MIM, Tinsley Industrial Park Shepcote Way Sheffield, S9 1TH, United Kingdom \\ ${ }^{e}$ Universidad de Castilla-La Mancha, ETSII-UCLM, Avda. Camilo José Cela s/n, \\ E-13071 Ciudad Real, Spain
}

\begin{abstract}
The paper deals with rheological properties of highly filled polymers used in powder injection molding (PIM) technology. Within the experimental framework 7 PIM feedstocks based on superalloy Inconel 718 powder were prepared. Each feedstock contains the fixed amount of powder loading and the same composition of binder system consisting of three components: polyethylene glycol (PEG), polymethylmetacrylate (PMMA) and stearic acid (SA). The scope was to carry out how PEG molecular weight would influence the feedstock flow properties, such as effect of temperature on viscosity, shear sensitivity and activation energy. Based on the results, the feedstock with PEG $(20000 \mathrm{~g} / \mathrm{mol})$ exhibited stable flow properties insted of using PEG (1 $500 \mathrm{~g} / \mathrm{mol}$ ) showing too low viscosity for injection molding process.
\end{abstract}

Keywords: powder injection molding, polyethylene glycol, molecular weight, rheology, activation energy

\section{INTRODUCTION}

PIM is a technological process which combines an injection molding with powder metallurgy and allows production of metal or ceramic components into the net complex shape [1]. Polymeric binder system provides suitable flow of the powder into the mold cavity. Once the part is injected, the binder is removed, preferably step-bystep. To attain the requirement of sequential debinding, the polymeric binder is usually a multi-component system [2]. The major component provides suitable flow properties and should be easily removed from the part in the first stage of debinding [3].

Nowadays, the PEG is a common part of partially-water soluble binder systems, because it can be easily removed from the injected components by immersion in water. This creates an interconnected network of pores from which the backbone polymer can escape during the final thermal step of debinding.

One of the most important aspects is the suitable binder composition, that will affects mostly the flow properties providing a successful injection molding without 
introducing defects. Various powder and binder systems have been investigated. However, only few papers focused especially on the effect of PEG molecular weight on flow properties $[4,5]$.

Shear viscosity $\eta$ is the most widely measured rheological properties because reflects the flow properties of given material. Nevertheless, the other rheological parameters such as activation energy could be also helpful in sense of better understanding the temperature sensitivity. For high shear rates which are typical during injection molding, the capillary rheometer is the most effective instrument for measuring shear viscosity.

In this work the flow properties of feedstocks based on superalloy Inconel 718 were investigated. The mixtures contained the fixed amount of powder and the same composition of the binder, where the major component PEG varied in molecular weight (from 1,500 to $20,000 \mathrm{~g} / \mathrm{mol}$ ). The backbone component was PMMA and a small amount of SA was also added to facilitate the lubrication. The scope was to carry out how the molecular weight of PEG can influence the viscosity curves, as well as the temperature and shear sensitivity of feedstocks.

\section{EXPERIMENTAL}

\section{Powder and binder characteristics}

\section{Powder}

The nickel-based superalloy Inconel 718 provided by Sandvik Osprey was used in this study. The particle size distribution was determined by a particle size laser analyser Malvern Mastersizer 3000 and the shape of particles was observed from scanning electron microscopy (SEM) using (Vega II LMU, Tescan). The obtained characteristics are shown in Fig. 1.
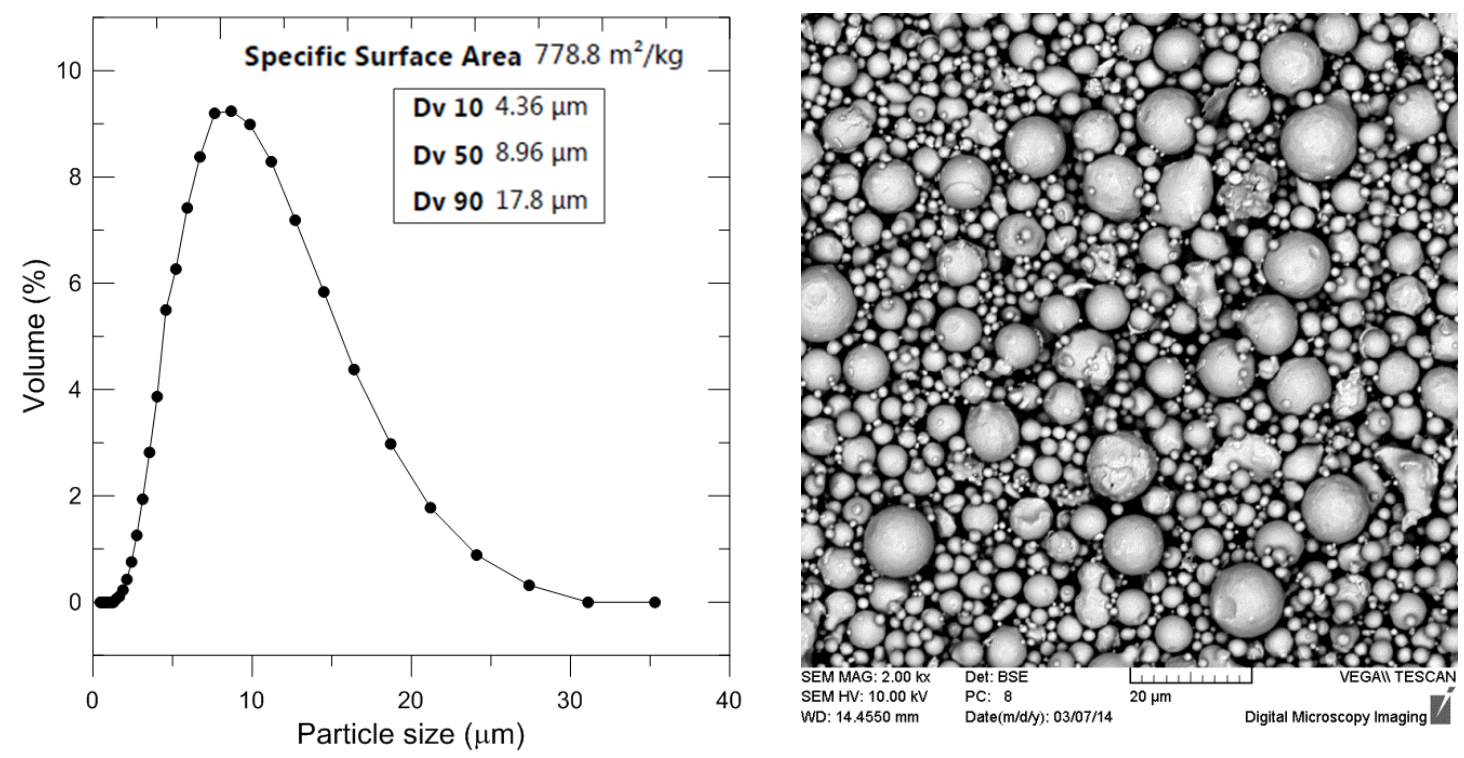

FIGURE 1. (a) Particle Size Distribution, (b) SEM micrograph of Inconel 718 powder 


\section{Binder}

The three-component binder system was developed in Sheffield University and its composition is listed in Table 1.

TABLE 1. Composition of a binder system

\begin{tabular}{lll}
\hline Component & Abbreviation & $\boldsymbol{w t} \%$ \\
\hline Major component & PEG & 83 \\
Backbone & PMMA & 15 \\
Surfactant & SA & 2 \\
\hline
\end{tabular}

The major component PEG provides low viscosity of feedstock and easy removal in the water, the backbone polymer PMMA ensures strength of an injected part, and a small amount of surfactant SA facilitates lubrication. PEG was supplied by Fluka and Sigma-Aldrich. The backbone polymer in form of emulsion with finely dispersed 0.1 $0.2 \mu \mathrm{m}$ PMMA particles was specially prepared by Scott Bader Co Ltd, Wellingborough (UK) and SA was supplied by Prolabo Chemicals (UK).

\section{Feedstock preparation}

Seven types of feedstocks were prepared with the fixed powder loading (59 vol. \%) and binder composition differing in molecular weight of PEG, as is shown in Table 2.

TABLE 2. Feedstocks varing in PEG $M_{w}$

\begin{tabular}{ll}
\hline Feedstock & $\mathbf{M}_{\mathbf{w}}(\mathrm{g} / \mathrm{mol})$ \\
\hline P1.5K & PEG 1500 \\
P4K & PEG 4000 \\
P6K & PEG 6 000 \\
P8K & PEG 8000 \\
P10K & PEG 10 000 \\
P12K & PEG 12 000 \\
P20K & PEG 20 000 \\
\hline
\end{tabular}

\section{Rheological measurement}

Rheological properties were measured using a capillary rheometer Goettfert 50 (Gemany). Viscosity dependence on shear rate at different processing temperatures $\left(120,140,160^{\circ} \mathrm{C}\right)$ was measured using the capillary die with $L / \mathrm{D}$ ratio of $20 / 1$. Then, end pressure effects were evaluated for all feedstocks at $140{ }^{\circ} \mathrm{C}$ by extrapolation of linear Bagley plots measured with 3 capillaries; having the constant diameter of $1 \mathrm{~mm}$ and lengths of 5, 10 and $20 \mathrm{~mm}$, with the entry angle of $90^{\circ}$. WeissenbergRabinowitsch correction was used to obtain the thrue shear rates and finaly, the corrected viscosites were compared to apparent viscosities. Activation energy was calculated according to Arrhenius equation. 


\section{Measured Data Processing}

Using a capillary die of radius $R$ the volumetric flow rate $\dot{Q}$ causes an apparent (Newtonian) wall shear rate:

$$
\dot{\gamma}_{a}=\frac{4 \dot{Q}}{\pi R^{3}} \quad \dot{Q}=S v
$$

where $S$ represents the area of the barrel and $v$ is velocity. By changing the piston speed the different shear rates to the sample are applied. The shear rate calculated from formula (1) is apparent, because it is valid only for Newtonian fluids.

The apparent wall shear stress $\tau_{a}$ is calculated from the measured pressure drop $\Delta p$ as follows:

$$
\tau_{a}=\frac{\Delta p R}{2 L}
$$

where $L$ is the length of the capillary die. In this case, the shear stress is apparent, because it is not taken into account the entrance and exit effects. In Figure 2 is shown, that obtained pressure from barrel $\Delta p$ is total pressure drop from the barrel to the capillary exit, $\Delta p_{c a p}$ is the pressure drop over the length of the capillary where the flow is fully developed, and $\Delta \mathrm{p}_{\mathrm{end}}=\Delta \mathrm{p}_{\mathrm{ent}}+\Delta \mathrm{p}_{\text {exit }}$ is the excess pressure, known as end pressure drop due to the entrance and exit flows [6].
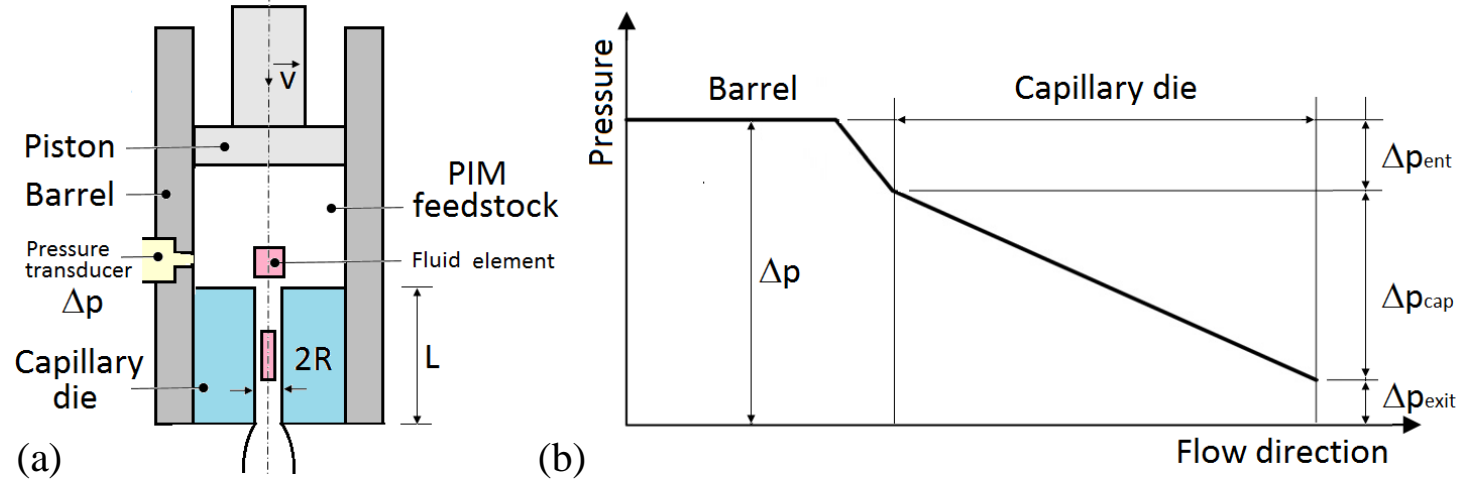

FIGURE 2. Schematic Diagram of (a) Capillary Rheometer, (b) Excess Pressure in Flow Direction

Then, the apparent viscosity $\eta_{a}$ can be obtained by:

$$
\eta_{a}=\frac{\tau_{a}}{\dot{\gamma}_{a}}
$$

The other important aspect of the rheological characterization for PIM feedstocks is the temperature dependence of viscosity [7], where relationship between viscosity $\eta$ and temperature $T$ can be expressed from Arrhenius equation:

$$
\eta=A \exp \left(\frac{E_{a}}{R T}\right)
$$


where $A$ is the pre-exponential factor, $E_{a}$ is the flow activation energy, $R$ is the gas constant and $T$ is the absolute temperature in K. Taking natural logarithm at both sides, Eq. (5) was obtained:

$$
\ln \eta=\ln A+\left(\frac{E_{a}}{R}\right) \frac{1}{T}
$$

According to Eq. (5), activation energy $E_{a}$ can be calculated from the slope of $\ln \eta$ vs. $1 / T$ at a certain shear rate.

\section{RESULTS AND DISCUSSION}

\section{Rheological investigation}

\section{Effect of PEG molecular weight on Flow Behavior}

The apparent viscosity curves of feedstocks measured at three different temperatures are shown in Figure 3. As can be seen, all feedstocks demonstrated pseudoplastic behavior in the range of shear rate from 35 to $25001 / \mathrm{s}$. It is also obvious, that with higher molecular weight of PEG component, the viscosity of feedstocks increase, as was expected. The viscosity of mixture P1.5K having the lowest $M_{w}$ PEG exhibited too low viscosity, which can cause an easily powder binder separation during injection process and thus create defects in the final sintered part.

It can be observed, that at higher shear rates, especially, at low temperature, most of the feedstocks tend to lower shear rate sensitivity and it seems, that they are closed to reach the $2^{\text {nd }}$ Newtonian plateau.

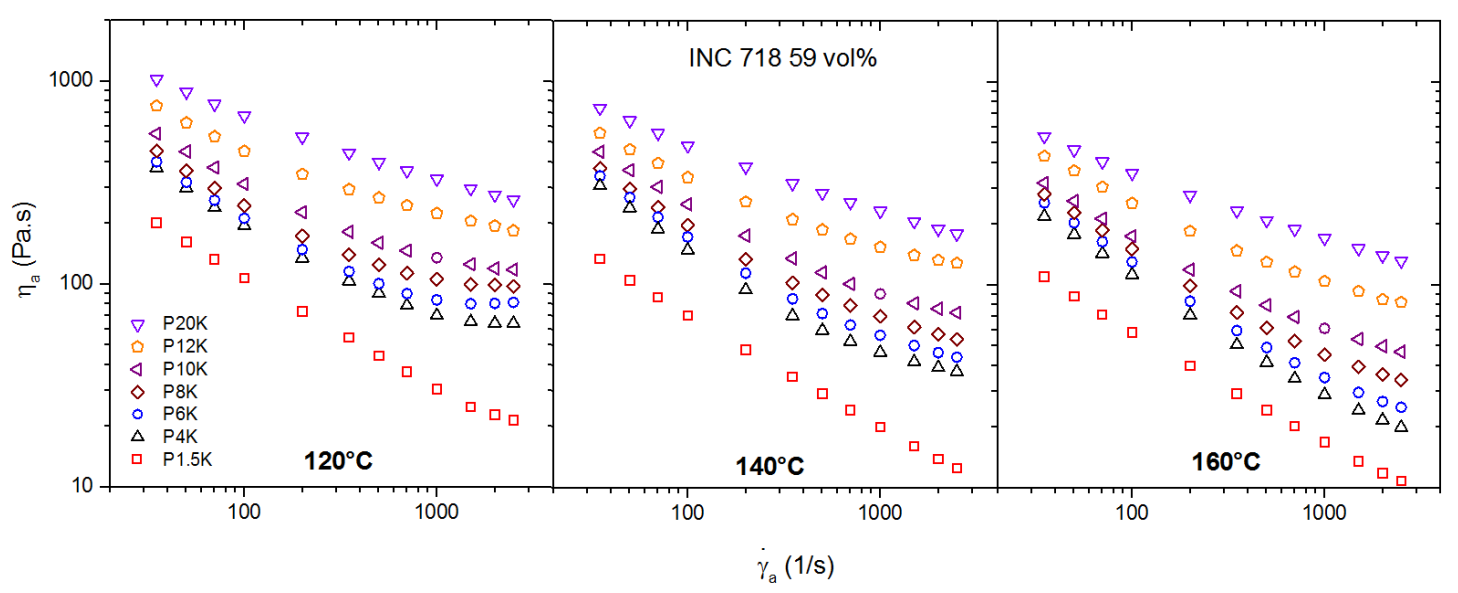

FIGURE 3. Feedstock Apparent Viscosity vs. Shear Rate

For pseudoplastic material, the correction of shear rate and shear stress should be done to obtain the real value of viscosity. 


\section{Bagley Correction}

The correction of wall shear stress due to end (entrance and exit) effects can be corrected by Bagley method; using a series of capillary dies with the same diameter $D$ but different lengths $L$.

Figure 4 (a) shows the plots of $\Delta p$ against $L / D$ for different constant shear rates of feedstock P6K at $140{ }^{\circ} \mathrm{C}$. Extrapolation of fitting lines to $L / D=0$, the end pressure $\Delta p_{\text {end }}$ can be subtracting from the $y$ axis.

Figure 4 (b) demonstrates the $\Delta p_{\text {end }}$ of all feedstocks as a function of shear rate. As can be seen, the $\Delta p_{\text {end }}$ linearly increase with higher shear rate, except the feedstock P1.5K which has the constant value. This could be caused due to a very low measured pressure and also by sensitivity of pressure transducer. The feedstock P12K achieved the $\Delta \mathrm{p}_{\text {end }} 14.3$ bar at shear rate $25001 / \mathrm{s}$, while the values for feedstock P1.5K did not reach 1 bar.
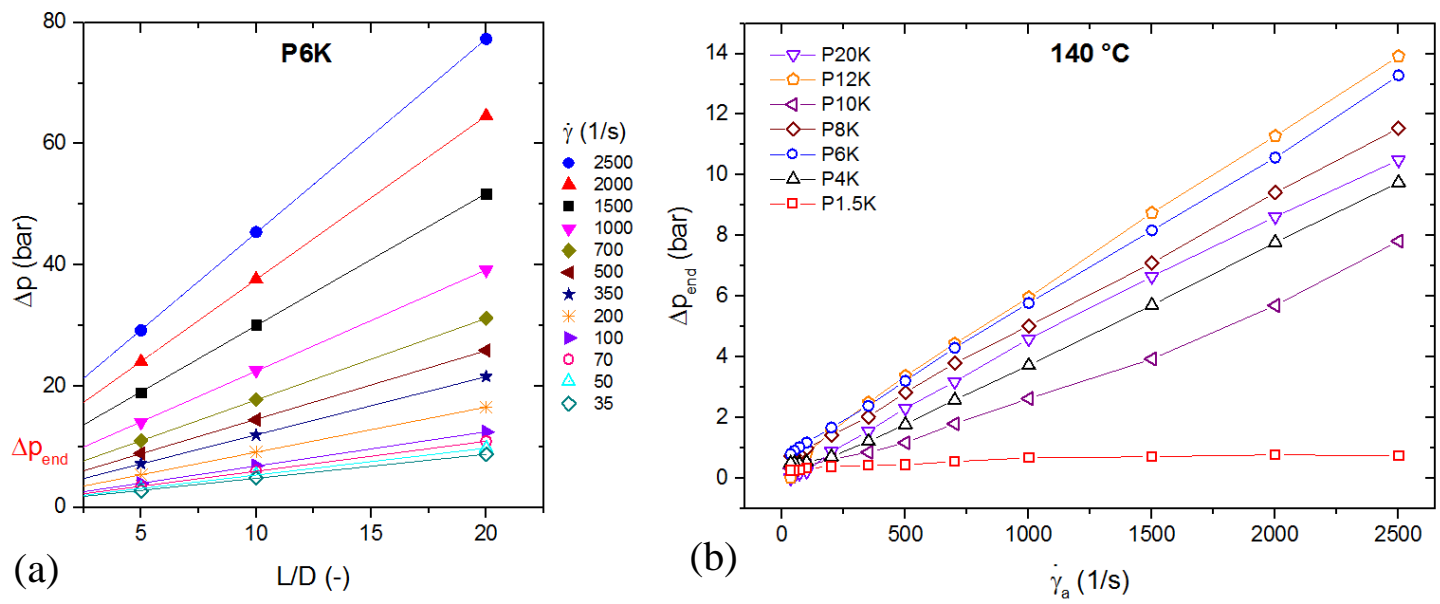

FIGURE 4. (a) Linear Bagley Plots of Feedstock P6K at $140^{\circ} \mathrm{C}$ to Determine end Pressure, (b) end Pressure of all Feedstocks as the Function of Apparent Shear Rate

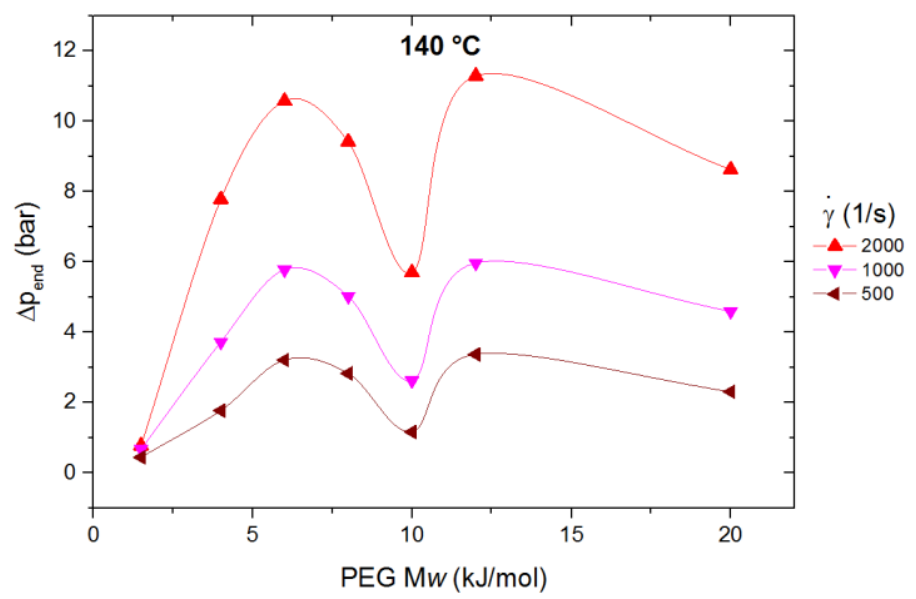

FIGURE 5. Complex Dependence of End Pressure on PEG Molecular Weight at Constant Shear Rate 
It is interesting, that the dependence of end pressure on PEG molecular weight at constant shear rate is not linear, but very complex, as is depicted in Figure 5. This may be explained due to different distribution of molecular weight of each PEG.

The corrected (true) wall shear stress $\tau_{c}$ can be calculated as follows:

$$
\tau_{c}=\frac{\left(\Delta p-\Delta p_{e n d}\right) R}{2 L}
$$

\section{Weissenberg-Rabinowitsch Correction}

The correction of apparent shear rate within a capillary die due to the non-parabolic velocity profile can be calculated, according to Rabinowitsch analysis [8], as follows:

$$
\dot{\gamma}_{c}=\dot{\gamma}_{a}\left(\frac{3 n+1}{4 n}\right)
$$

where $n$ is the non-Newtonian index, which can be obtained from a log-log plot of $\tau_{\mathrm{c}}$ vs. $\dot{\gamma}_{a}$. If the obtained curve is straight, the slope is constant non-Newtonian index. Otherwise, if the line is not straight, as can be seen in Figure 6 (a), a polynomial can be used to fit data [9]:

$$
\log \left(\tau_{c}\right)=a \log ^{2}\left(\dot{\gamma}_{a}\right)+b \log \left(\dot{\gamma}_{a}\right)+c
$$

From the polynomial, Eq. (7) we can calculate the needed derivative:

$$
n=\frac{d \log \left(\tau_{c}\right)}{d \log \left(\dot{\gamma}_{a}\right)}=2 a \log \left(\dot{\gamma}_{a}\right)+b
$$

Then, the non-Newtonian index is calculated from the tangent in every point on the line. For all feedstocks, the index $n$, representing shear sensitivity, varied from 0.8 to 0.2 in the shear rate range, as shown in Figure 6. (b). The highest shear sensitivity was obtained at low shear rate, while at higher shear rates the values of $n$ increased.
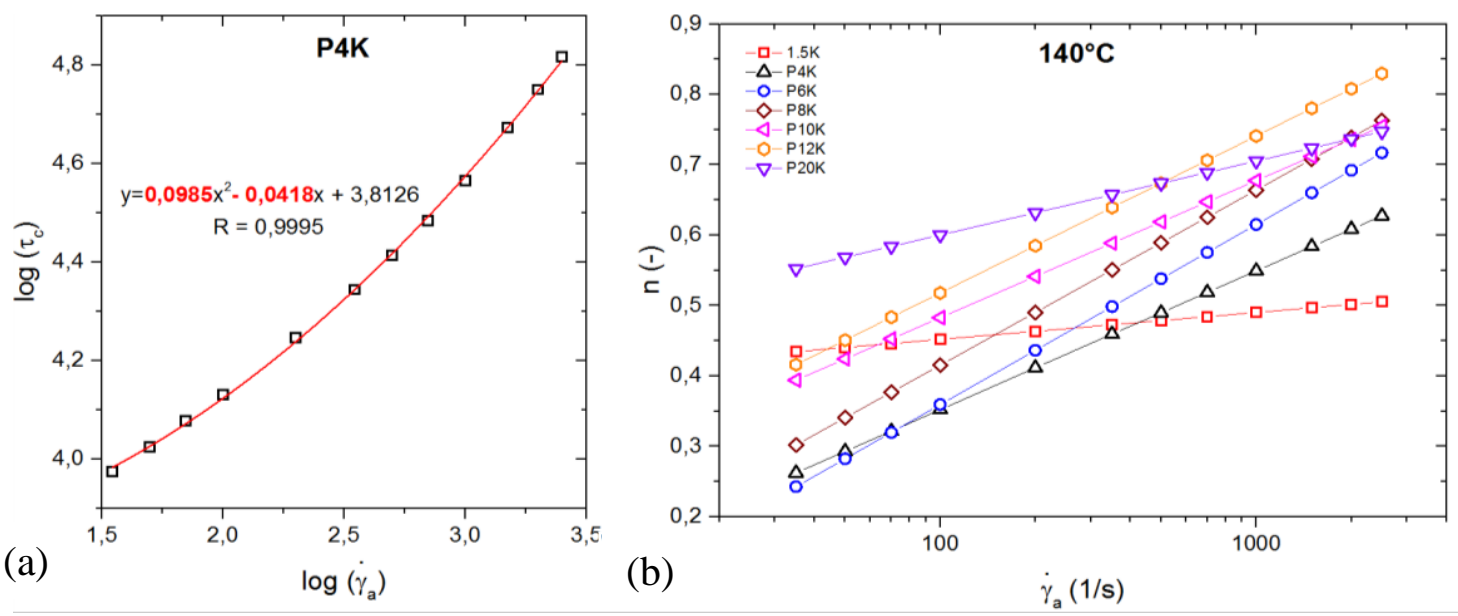

FIGURE 6. (a) Polynomial fitting of feedstock $\mathrm{P} 4 \mathrm{~K}$ at $140^{\circ} \mathrm{C}$, (b) comparison of non-Newtonian index vs. apparent for each feedstock 


\section{Corrected Viscosity}

Once the apparent shear rate was corrected to the true shear rate, then corrected viscosity $\eta_{c}$ could be calculated as:

$$
\eta_{c}=\frac{\tau_{c}}{\dot{\gamma}_{c}}
$$

Figure 7. demonstrates differences between the apparent and corrected viscosity as a function of shear rate. As can be seen, the values of corrected viscosities are smaller than apparent viscosities. The correction is strongly recommended, in the case the data is to be used for design, flow simulation or fundamental studies. On the contrary, apparent viscosity can be used to compare different samples tested with the same instrument, die and known flow properties.

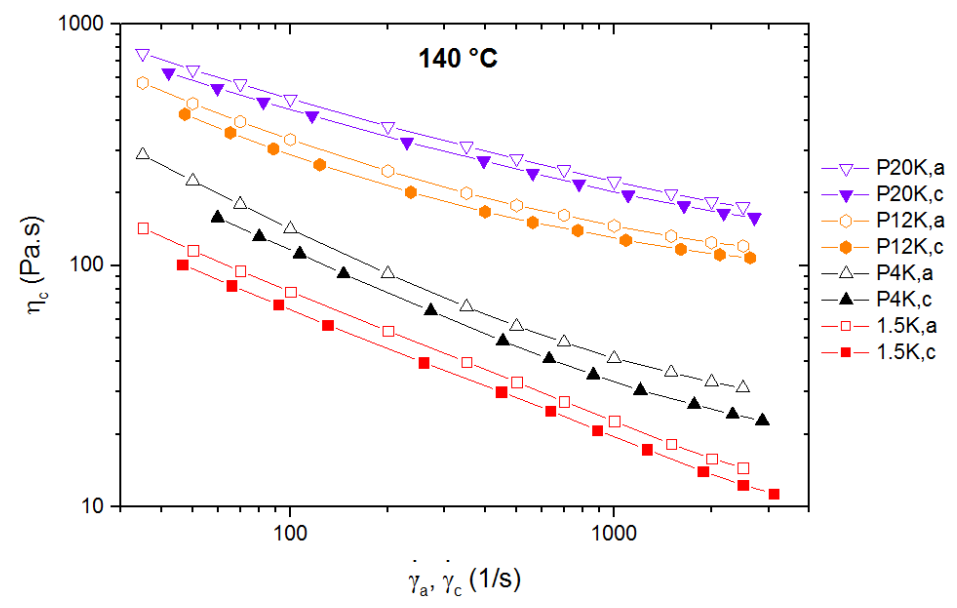

FIGURE 7. Comparison of apparent and corrected viscosity vs. shear rate of four feedstocks at $140{ }^{\circ} \mathrm{C}$

\section{Activation Energy}

Activation energy indicates the temperature sensitivity of feedstocks and due to comparative character; data used for this evaluation was without correction. Figure 8. (a) demonstrates plots according Eq. (5) and as was mentioned above, from the slope of linear fitting, the flow activation energy measured at constant shear rate can be obtained.

In Figure 8. (b), the results showed that tendency for most of the feedstocks with increasing PEG molecular weight, the activation energy will decrease; the exception was the feedstock P1.5K.

The same trend was obtained by W.-W. Yan et al. [4], they examined 4 different feedstocks based on alumina also with different PEG molecular weight, but they measured activation energy only at the shear rate of $3001 / \mathrm{s}$, while injection molding occurs at much higher values.

The lowest activation energy was obtained for the feedstock P20K; except the feedstocks P1.5K which had too low viscosity for injection molding. 

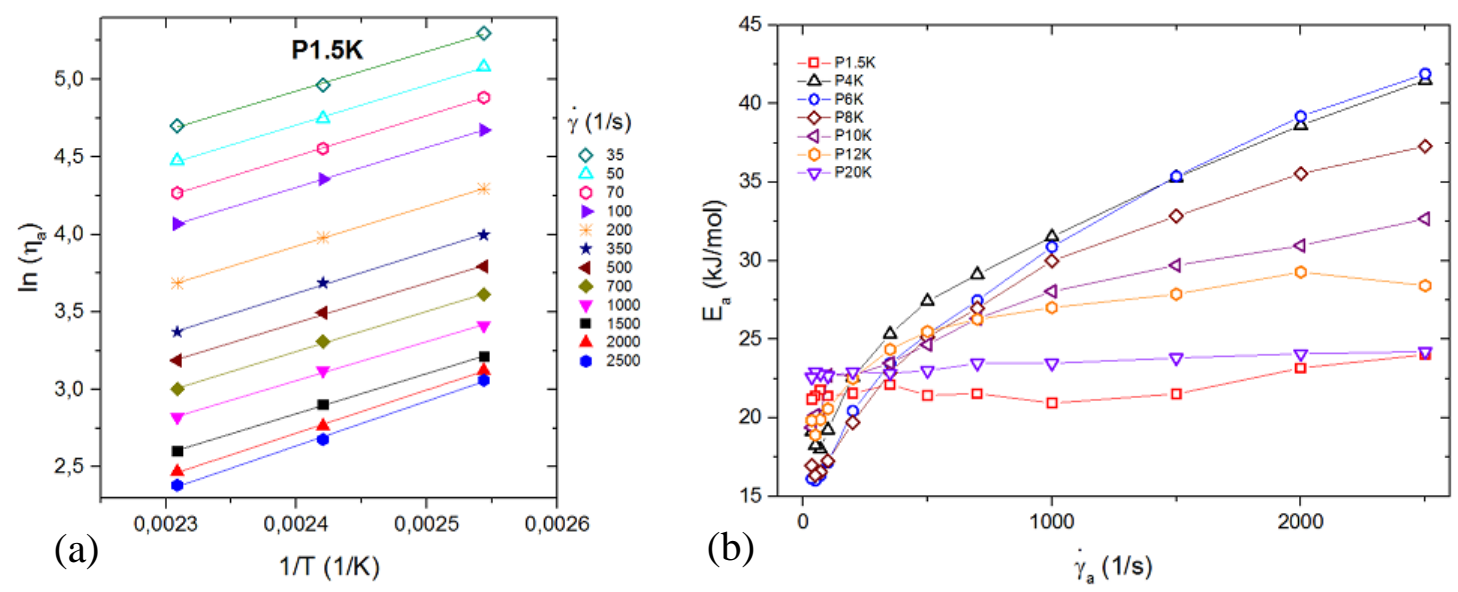

FIGURE 8. (a) Arrhenius Plots of Feedstock P1.5K (b) Activation Energy of All Feedstocks

In addition the $E_{a}$ of the feedstock $\mathrm{P} 20 \mathrm{~K}$ did not change apparently with the shear rate and remains almost constant in the shear rate range. Then, this feedstock indicates the smallest sensitivity of viscosity to temperature and will be the most suitable for injection molding from processing point of view in wide temperature range. The high values of $E_{a}$ will cause the faster viscosity increase during cooling, which can introduced internal stresses.

\section{CONCLUSION}

The obtained results showed that molecular weight of PEG component in highly filled compounds will influence the flow properties. All mixtures demonstrated pseudoplastic behavior in the measured shear rate range. It was found, that shear viscosity increased with higher PEG molecular weight. Viscosity of the feedstock P1.5K was extremely low, which can cause an easily powder binder separation and introduce defects in the final sintered parts. The end pressure (entrance and exit effects) obtained from Bagley plots demonstrated linear increasing with higher shear rate. The non-Newtonian index $n$, representing shear sensitivity varied from 0.8 to 0.2 at $140{ }^{\circ} \mathrm{C}$. All feedstocks, at higher shear rates, tended to the lower shear rate sensitivity. In case, the data is to be used for flow simulation, the correction of viscosity is strongly recommended. From processing point of view, the feedstock P20K seems to be the most suitable for the injection molding; its activation energy was the lowest and almost constant in the measured shear rate range.

\section{ACKNOWLEDGMENTS}

Operational Program Research and Development for Innovations co-funded by the ERDF and national budget of Czech Republic, within the framework of project Centre of Polymer Systems (reg. number: CZ.1.05/2.1.00/03.0111) is acknowledged for financial support. This study was also supported by the internal grant of TBU in Zlín IGA/FT/2015/001 funded from the resources of specific university research. 


\section{REFERENCES}

1. R. M. German and A. Bose, Injection molding of metals and ceramics: Metal Powder Industries Federation, Priceton, (1997).

2 D. F. Heaney, 2012, Handbook of metal injection molding: Woodhead Publishing, (2012).

3. G. P. Chen, G. W. Cao, N. Edmonds, Debinding behaviour of a water soluble PEG/PMMA binder for Ti metal injection moulding. Mat. Chem. and Phys. 139, 557-565 (2013).

4. W-W. Jang, K-Y. Jang, M-H. Hon, Effects of PEG molecular weights on rheological behaviour of alumina injection molding feedstock, Mat. Chem. and Phys. 78, 416-424 (2002).

5. M. D. Hayat, G. Wen, M. F. Zulkifli, P. Cao. Effect of PEG molecular weight on rheological properties of Ti-MIM feedstocks and water debinding behaviour. Powder Technology, 270, 296-301 (2015).

6. Mitsoulis and S. G. Hatzikiriakos, Bagley correction: the effect of contraction angle and its prediction. Rheol. Acta. 42, 309-320 (2003).

7. T. Honek, B. Hausnerova, P. Saha. Temperature Dependent Flow Instabilities of Highly Filled Polymer Compounds. Applied Rheology, 12 (2), 72-80 (2002).

8. P. J. Carreau, D. De Kee, R. P. Chhabra, Rheology of Polymeric Systems. Hanser, Munich (1997).

9. M. Zatloukal (private notes). 